\title{
Tensor Network Simulation of Non-Markovian Dynamics in Organic Polaritons
}

\author{
Javier del Pino, ${ }^{1}$ Florian A. Y. N. Schröder, ${ }^{2}$ Alex W. Chin, ${ }^{3,2}$ Johannes Feist, ${ }^{1,}$ and Francisco J. Garcia-Vidal ${ }^{1,4, \dagger}$ \\ ${ }^{1}$ Departamento de Física Teórica de la Materia Condensada and Condensed Matter Physics Center (IFIMAC), \\ Universidad Autónoma de Madrid, E-28049 Madrid, Spain \\ ${ }^{2}$ Cavendish Laboratory, University of Cambridge, J. J. Thomson Avenue, Cambridge, CB3 OHE, United Kingdom \\ ${ }^{3}$ Institut des NanoSciences de Paris, Sorbonne Université, 4 place Jussieu, boîte courrier 840, 75252, Paris Cedex 05, France \\ ${ }^{4}$ Donostia International Physics Center (DIPC), E-20018 Donostia/San Sebastián, Spain
}

(Received 12 April 2018; published 26 November 2018)

\begin{abstract}
We calculate the exact many-body time dynamics of polaritonic states supported by an optical cavity filled with organic molecules. Optical, vibrational, and radiative processes are treated on an equal footing employing the time-dependent variational matrix product states algorithm. We demonstrate signatures of non-Markovian vibronic dynamics and its fingerprints in the far-field photon emission spectrum at arbitrary light-matter interaction scales, ranging from the weak to the strong coupling regimes. We analyze both the single- and many-molecule cases, showing the crucial role played by the collective motion of molecular nuclei and dark states in determining the polariton dynamics and the subsequent photon emission.
\end{abstract}

DOI: 10.1103/PhysRevLett.121.227401

Organic polaritons, formed upon hybridization of optical electromagnetic (EM) modes and Frenkel excitons of organic molecules [1-5], exist as a threefold mixture of photonic, electronic, and phononic excitations. As a result, modified nuclear effects in polaritons leads to tailored material [6-8] and chemical [9-13] properties. In this regard, experiments have underlined the impact of vibronic states in the polariton time evolution, with examples in thermalization and cooling of room $T$ condensates [14-16], thresholds in organic lasers [17,18], and lifetimes [19-21]. First studies on the impact of vibrations on organic polariton dynamics relied on the Fermi's golden rule within a Markovian approach [22,23], which neglects correlations and interplay between electronic and vibrational excitations. More recently, the influence of vibronic states in the spectroscopic properties of these systems [24,25] has been analyzed using the Holstein-Tavis-Cummings (HTC) model, in which the complex spectral density of the vibronic modes in organic molecules is modeled by a single phonon mode.

In this Letter we simulate the temporal dynamics of the polaritons supported by an optical microcavity filled with an ensemble of prototypical organic molecules (Rhodamine 800), as sketched in Fig. 1(a). In our approach, nuclear, electronic, and photonic processes are treated fully and on equal footing by employing the time-dependent variational matrix product states (TDVMPS) algorithm, which can provide quasiexact solutions of open quantum system dynamics by truncating the maximum entanglement between different system components [26-30]. Our fundamental study reveals a variety of regimes for the time evolution of the polariton populations, which experience vibration-assisted dynamics that translate into distinct far-field emission fingerprints. We observe clear signatures of non-Markovian behavior in the whole range of lightmatter coupling, both for single and multiple molecules coupled to an EM cavity mode. We find that the full

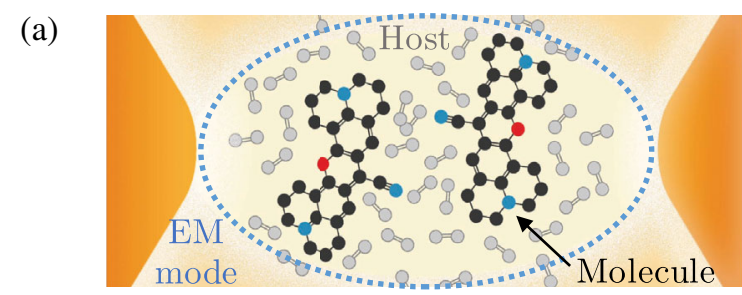

(b)

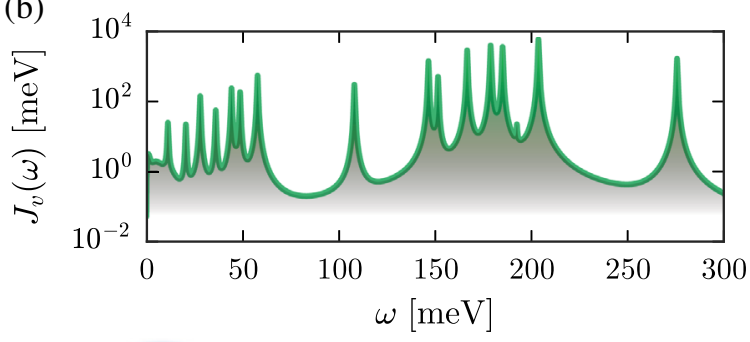

(c)

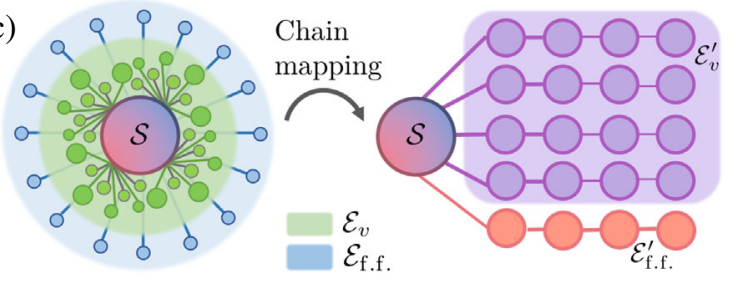

FIG. 1. (a) Sketch of a molecular ensemble interacting with a confined EM resonance (dashed region) and with the host environment (grey circles). (b) Vibrational spectral density for Rhodamine 800 molecule. (c) Scheme illustrating the mapping of the vibrational modes yielding the multichain Hamiltonian used in the simulations. 
vibrational structure of the molecules has to be treated for a complete understanding of the interplay between excitons, phonons, and photons.

Our model includes a collection of $N$ identical molecules with two electronic states (frequency $\omega_{e}$ and ladder operators $\hat{\sigma}_{ \pm}^{(i)}, i \in[1, \ldots, N]$ ), placed within the volume of a nano- or microcavity supporting a single dispersionless EM mode (frequency $\omega_{O}=\omega_{e}=2.5 \mathrm{eV}$ ), with annihilation operator $\hat{a}$. The total Hamiltonian contains three different parts, as schematically depicted in Fig. 1(c). First, the "bare" system $\mathcal{S}$ accounts for the excitons within the molecules, the cavity EM mode, and their coupling, measured by the collective Rabi frequency $\Omega_{R}$ and treated within the rotating wave approximation (setting $\hbar=1$ ),

$\hat{H}_{\mathcal{S}}=\omega_{O} \hat{a}^{\dagger} \hat{a}+\sum_{i=1}^{N} \omega_{e} \hat{\sigma}_{+}^{(i)} \hat{\sigma}_{-}^{(i)}+\frac{\Omega_{R}}{2 \sqrt{N}} \sum_{i=1}^{N}\left(\hat{a}^{\dagger} \hat{\sigma}_{-}^{(i)}+\hat{\sigma}_{+}^{(i)} \hat{a}\right)$.

Direct dipole-dipole interactions at typical intermolecular distances in strong-coupling experiments only provide small corrections [31-33] and are thus neglected here for simplicity and generality. In the single-excitation subspace, $\hat{H}_{\mathcal{S}}$ is exactly solvable, with two types of eigenstates: (i) upper polariton (UP) and lower polariton (LP) $| \pm\rangle=\left(\hat{a}^{\dagger}|G\rangle \pm\right.$ $|B\rangle) / \sqrt{2}$ with frequencies $\omega_{ \pm}=\omega_{O} \pm \Omega_{R} / 2$, which result from the hybridization of the collective excitonic bright state $|B\rangle=\left(\sum_{i=1}^{N} \hat{\sigma}_{+}^{(i)}|G\rangle\right) / \sqrt{N}$ with the cavity EM mode (with $|G\rangle$ the global vacuum state) and (ii) $N-1$ purely excitonic dark states (DSs), $|d\rangle \in \mathcal{D}$, orthogonal to $|B\rangle$, with frequency $\omega_{e}$.

The second part of the Hamiltonian describes the vibrational subspace $\mathcal{E}_{v}^{(i)}$ containing $M_{v}$ vibrational modes for each molecule, and their elastic coupling to the excitons. The $k$ th vibrational mode is approximated by a harmonic oscillator of frequency $\omega_{k}$ (valid close to the equilibrium position) with annihilation operator $\hat{b}_{k}^{(i)}$ and $k$-dependent exciton-phonon coupling strength $\lambda_{k}^{(i)}$,

$\hat{H}_{v}=\sum_{i=1}^{N} \sum_{k=1}^{M_{v}} \omega_{k} \hat{b}_{k}^{\dagger(i)} \hat{b}_{k}^{(i)}+\sum_{i=1}^{N} \sum_{k=1}^{M_{v}} \lambda_{k}^{(i)}\left(\hat{b}_{k}^{(i)}+\hat{b}_{k}^{\dagger(i)}\right) \hat{\sigma}_{+}^{(i)} \hat{\sigma}_{-}^{(i)}$.

The properties of these modes, $\left\{\omega_{k}, \lambda_{k}^{(i)}\right\}$, are encoded in the spectral density, assumed to be identical for all molecules, $J_{v}^{(i)}(\omega)=J_{v}(\omega)=\pi \sum_{k=1}^{M_{v}} \lambda_{k}^{2} \delta\left(\omega-\omega_{k}\right)$. For Rhodamine 800 this density is extracted from the spectroscopic measurements in Ref. [34], as displayed in Fig. 1(b), with vibrational frequencies located in the range $[0,0.3] \mathrm{eV}$, and reorganization energy $\Delta=\int_{0}^{\infty}\left\{\left[J_{v}(\omega)\right] / \pi \omega\right\} \mathrm{d} \omega \approx$ $112 \mathrm{meV}$. While no interactions between vibrational modes are included in the model, internal vibrational decay due to interactions with the host medium is partially represented through the nonzero width of the peaks in $J_{v}(\omega)$. Furthermore, we have checked that the results presented below do not depend sensitively on the properties of $J_{v}(\omega)$.

The third part of the Hamiltonian describes radiative far-field photon modes $\hat{f}_{l}$ and their coupling to the cavity EM mode, $\hat{H}_{r}=\sum_{l} \omega_{l} \hat{f}_{l}^{\dagger} \hat{f}_{l}+\eta_{l}\left(\hat{a}^{\dagger} \hat{f}_{l}+\hat{f}_{l}^{\dagger} \hat{a}\right)$ [35]. In a way similar to vibrational modes, we introduce here the spectral density for the photonic subspace, $\mathcal{E}_{r}$, as $J_{r}(\omega)=$ $\pi \sum_{l} \eta_{l}^{2} \delta\left(\omega-\omega_{l}\right)=\kappa \omega^{3} /\left(2 \omega_{O}^{3}\right)$. We set the bare-cavity decay rate $\kappa=2 J_{r}\left(\omega_{O}\right)$ to $50 \mathrm{meV}$, typical for plasmonic or dielectric cavities.

For typical molecules, the large number of vibrational modes, $M_{v} \sim 10^{2}$, makes direct diagonalization of the total Hamiltonian infeasible. We resolve this by applying the TDVMPS approach. We first perform an orthogonal chain mapping of the modes in the $N$ vibrational (green) and the free-space photon (blue) environments $\left(\mathcal{E}_{v}^{(i)}\right.$ and $\mathcal{E}_{r}$ ), sketched in Fig. 1(c) and detailed in Ref. [36], regrouping them in chains with length $L=M_{v}$ with nearest-neighbor hopping, with only the first chain mode coupled to the exciton-photon subspace $\mathcal{S}$ (red-blue) [43]. The wave function $|\psi(t)\rangle$ is represented by a tensor network [44] with maximum bond dimensions $D$ with a structure mimicking the transformed Hamiltonian. If a single root tensor stores the system $\mathcal{S}$, its size scales exponentially with $N$, leading to a severe memory bottleneck. This scaling can be efficiently reduced while maintaining precision by decomposing $|\psi(t)\rangle$ into a tree tensor network, with a structure determined to minimize the entanglement between nodes $[45,46]$, and each final branch coupled to a single chain (see Ref. [36] for details). This allows for the treatment of $N=16$ molecules coupled to $N+1$ environments with $L=350$ modes each, i.e., a system for which the full Hilbert space has a dimension of $\approx 50^{(N+1) L}$ (allowing 50 basis states per phonon mode), with $(N+1) L=5950$, through a wave function described by $\approx 10^{8}$ parameters. To further ameliorate memory issues for large chain mode occupations, we employ an optimal boson basis for the chain tensors, determined on the fly [47]. A more detailed description of the theoretical approach can be found in Refs. [48,49]. We here focus on the time evolution after excitation, but note that the same approach also allows efficient calculation of the "lower polaron-polariton" [11,25,50,51], as shown in Ref. [49].

We first explore single-molecule strong coupling ( $N=1$, for which there are no dark states), recently realized in plasmonic nanocavities [52,53]. We set the initial state $(t=0)$ to the bare (vibrationally undressed) UP, $|+\rangle$, as would be produced by an on-resonance laser pulse short enough to ensure that nuclear motion can be neglected during its action. This allows us to restrict the simulation to only the zero- and single-excitation subspaces of $\hat{H}_{\mathcal{S}}$. Figure 2 shows the time evolution of the populations 


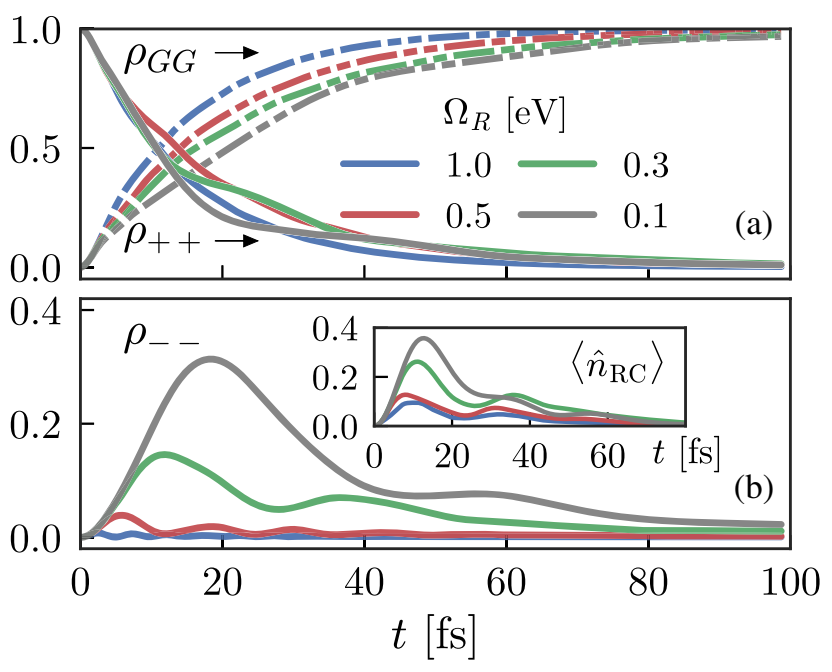

FIG. 2. Dynamics of reduced density matrix populations for a single molecule as a function of the Rabi frequency $\Omega_{R}$, including (a) vacuum and upper polariton states and (b) the lower polariton. The inset in (b) shows the occupation of the collective reaction coordinate in the vibrational environment.

$\left\{\rho_{G G}, \rho_{ \pm \pm}\right\}$, extracted from the system density matrix $\hat{\rho}_{\mathcal{S}}(t)=\operatorname{Tr}_{\mathcal{E}_{v}, \mathcal{E}_{r}}\{|\psi(t)\rangle\langle\psi(t)|\}$. Details of the numerical parameters and convergence behavior can be found in the Supplemental Material [36]. When $\Omega_{R}$ [=1 eV in Fig. 2(a)] is much larger than the vibrational frequencies $\left(\omega_{v}^{\text {cut }}=0.3 \mathrm{eV}\right)$, the bare UP $\left(\rho_{++}\right)$shows rapid exponential decay to the ground state $\left(\rho_{\mathrm{GG}}\right)$ through photon emission, with a negligible population increase of the LP. This is exactly what an approach treating $\mathcal{E}_{v}$ and $\mathcal{E}_{r}$ as Markovian (memory-less) baths would predict, as there are no available phonon modes that could induce transitions between an UP and LP. However, already for the case $\Omega_{R}=0.5 \mathrm{eV}$, our results show a new decay pathway for the UP, which can relax through phonon emission to the LP. A closer look reveals that the emission of photons, $\rho_{++} \rightarrow \rho_{G G}$, is superimposed to a coherent exchange of population between the UP and LP [see Fig. 2(b)]. Moreover, this dynamics is accompanied by a collective excitation of the vibrational modes, as displayed by the time evolution of the reaction coordinate population, $\hat{b}_{\mathrm{RC}}=\sum_{k} \lambda_{k} \hat{b}_{k} / \sqrt{\sum_{k} \lambda_{k}^{2}}$ [inset of Fig. 2(b)]. Neither of these effects could be reproduced by a Markovian approximation, which would lead to single-phonon transitions between polaritons at rates $\Gamma_{i f}^{v}=2 J_{v}\left(\omega_{i f}\right)$, where $\omega_{i f}=$ $\omega_{i}-\omega_{f}$ is the transition frequency. As the Rabi splitting is larger than the largest vibrational frequency available, decay into the LP is forbidden within the Markovian limit [54]. The amplitude of these oscillations is enhanced for lower Rabi frequencies, where vibronic and photonic couplings become comparable [see cases $\Omega_{R}=0.3 \mathrm{eV}$ and $\Omega_{R}=0.1 \mathrm{eV}$ in Fig. 2(b)]. Here the onset of nonexponential behavior in both $\rho_{++}$and $\rho_{G G}$ is noticed, resulting in delayed photon emission. In particular, while the UP starts emitting photons immediately, the LP population is maximized after approximately one cycle of coherent oscillation of the reaction coordinate before radiative decay starts to dominate.

We next proceed to discuss the many-molecule case, in which dark states have a severe impact on the dynamics [55]. In this section we compare our results with those emerging from a standard master equation derived using the Markovian Bloch-Redfield-Wangsness (BRW) approach [56,57], which considers solely the value of $J_{v}(\omega)$ at the transition frequency within the so-called secular approximation and is restricted to single-phonon transitions. We note that while it is possible to derive more advanced Markovian and non-Markovian master equations [58-60], BRW theory already goes significantly beyond the widely employed Lindblad master equation approach, and allows clear identification of non-Markovian and multiphonon processes in the TDVMPS simulations.

For very large $\Omega_{R}$ [see Fig. 3(a)], the UP decays mostly by photon emission. While vibrational decay to the LP is negligible, some population does reach the DSs (with $\left.\rho_{\mathcal{D D}}=\sum_{d} \rho_{d d}\right)$. Remarkably, while the photonic decay can be accurately determined by BRW theory, the prediction for the DSs pathway disagrees with the TDVMPS calculation. BRW theory predicts $\rho_{\mathcal{D D}}=\rho_{--}=0$ as there are no phonon modes at the required transition frequencies, while the TDVMPS calculation shows that the DS reservoir is indeed populated. This demonstrates that vibrationdriven decay from polaritonic states [22,61-63] can occur efficiently even when no vibrational modes are resonant with the transition frequency, and that the decoupling from vibrational modes that is found for the LP under collective strong coupling $[10,11,25,49,64]$ does not prevent decay of the UP.

For smaller values of the Rabi splitting, the UP-DS and DS-LP transition frequencies lie within the range of $J_{v}(\omega)$. Figure 3(b) shows that for $\Omega_{R}=0.5 \mathrm{eV}$, coherent population transfer to the DSs competes with the fast photonic decay of the UP, inducing threefold oscillations $\rho_{++} \leftrightarrow$ $\rho_{\mathcal{D D}} \leftrightarrow \rho_{--}$that persist over more than 50 fs. For a larger number of molecules, the population is "trapped" more efficiently in $\mathcal{D}$ and subsequently decays to the LP over several hundred femtoseconds before being emitted (see Ref. [36] for a comparison with the case $N=4$ ). While the intrinsic lifetimes of the LP and UP are similar due to efficient photon leakage out of the cavity, as seen in simulations initialized in the LP (not shown), the refilling from the DSs leads to its population persisting over much longer timescales. This observation agrees with the longtime tails observed in strongly coupled $J$ aggregates $[19,65]$. As the UP-DS-LP transition frequencies are not resonant with any vibration in the system [cf. Fig. 1(b)], the DSs and LP remain unpopulated within BRW theory. In contrast, TDVMPS method can represent both 

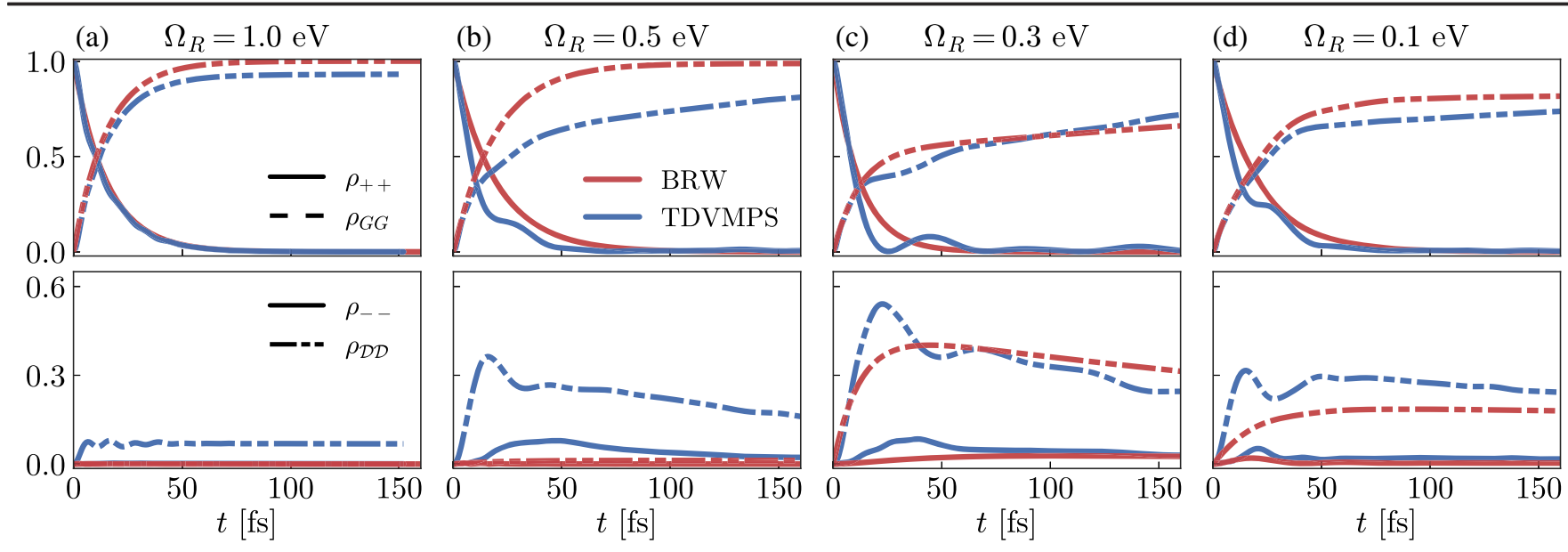

FIG. 3. Panels (a-d) depict the population dynamics for $N=16$ and several Rabi frequencies, with values shown in the titles. The occupations $\rho_{G G}, \rho_{++}$are displayed in the upper panels, while $\rho_{--}, \rho_{\mathcal{D D}}$ is shown in the lower ones, with distinctive line styles. Different colors depict the full numerical TDVMPS result and the Markovian limit calculated as described in the text.

multiphonon relaxation and the broadening of the polaritons due to decay, reducing the stringency of vibrational resonance conditions.

Similarly to the single-molecule case, vibrationally driven oscillations take longer to relax for smaller Rabi frequencies. Interestingly, the numerical agreement between Markovian and non-Markovian approaches is improved in the particular case of $\Omega_{R}=0.3 \mathrm{eV}$ [Fig. 3(c)], where the UP-DS transition is quasiresonant with a vibrational resonance [cf. Fig. 1(c)]. In this case, while the long-time behavior is reasonably well approximated by BRW theory, the rapid short-time oscillatory dynamics is averaged out. For even smaller Rabi frequencies, we do not observe a monotonic increase of the LP population, as opposed to the case $N=1$. In particular, Fig. 3(d) displays the case $\Omega_{R}=0.1 \mathrm{eV}$, where the UP population is only slightly more efficiently transferred to DSs and LP than for $\Omega_{R}=0.5 \mathrm{eV}$ [Fig. 3(b)]. Here the large phonon coupling leads to a rapid destruction of polariton coherence, i.e., loss of strong coupling [4].

In the Supplemental Material [36], we additionally compare TDVMPS calculations with the single-mode HTC model, which has been successfully used to predict non-Markovian dynamics and energy transfer between exciton polaritons and DSs [11,24,50,66,67]. While it reproduces the dynamics in the first few femtoseconds (dominated by the reaction coordinate response) reasonably well, it consistently overestimates the coherent oscillations observed for $t \gtrsim 10 \mathrm{fs}$. In particular, it fails to correctly predict the excitation trapping in $\mathcal{D}$ and instead leads to enduring oscillations.

In typical organic polariton experiments, the collected farfield photons are the main source of available information. Specifically, short-time energy redistribution between polaritons could be traced by ultrafast pump-probe experiments $[19,68-70]$. The final part of this work is thus devoted to the fingerprints displayed in the time-resolved emission spectrum arising from the different dynamics regimes that have been analyzed above. Thanks to the full access to the reservoir degrees of freedom provided by TDVMPS framework [30], emission can be retrieved from the occupation of the far-field modes $\left\langle\hat{n}_{r}\left(\omega_{l}, t\right)\right\rangle=\left\langle\psi(t)\left|\hat{f}_{l}^{\dagger} \hat{f}_{l}\right| \psi(t)\right\rangle$.

For very large Rabi splittings $\left(\Omega_{R}=1 \mathrm{eV}\right)$, Fig. 4(a) shows dominant photonic emission from the UP, with a series of side lobes converging to the main emission line due to the coherent buildup of population in the free-space mode [71]. At timescales comparable to the reaction coordinate dynamics $\left(\tau_{\mathrm{RC}}=2 \pi / \omega_{\mathrm{RC}} \simeq 27 \mathrm{fs}\right)$, population transfer through the DSs reservoir reaches the LP and its emission is observed as well, as clearly seen in cuts at the bare polariton frequencies $\omega_{ \pm}$[lower panel of Fig. 4(a)] that

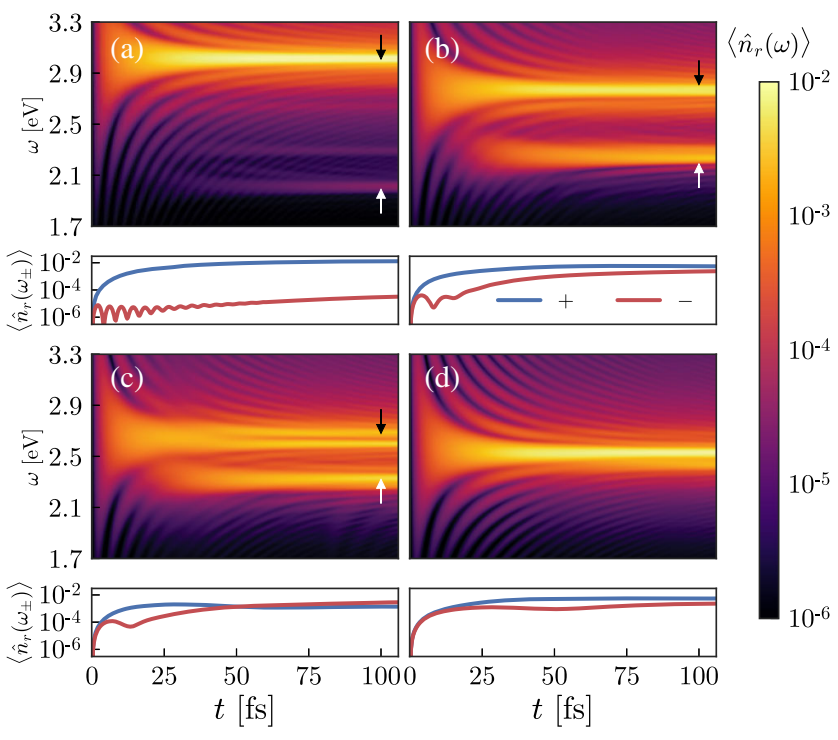

FIG. 4. Far-field photonic population as a function of frequency and time, for the same Rabi splittings as in Fig. 3 and $N=4$. The black and white arrows in panels (a)-(c) point to the approximate location of the bare UP and LP, merging into a single peak in panel (d). Each panel includes, in the lower parts, cuts at $\omega_{ \pm}$. 
display the buildup of far-field occupation. The asymptotic growth of the photons emitted by the LP continues after the UP is practically depopulated, due to the continued refilling from the dark states. In addition to the vibration-free polaritons $| \pm\rangle$, emission bands at intermediate energies are visible in the spectrum, in particular, at the baremolecule emission frequency $\omega_{e}-2 \Delta \approx 2.276 \mathrm{eV}$ in all cases. This is interpreted as due to small cavity admixtures to (vibrationally relaxed) dark states, in line with experimental observations $[65,72]$.

As the Rabi frequency is diminished, more efficient coupling from the UP to the DSs increases population transfer and induces brighter emission from the LP and its vibronic sidebands. For $\Omega_{R}=0.3 \mathrm{eV}$, depicted in Fig. 4(c), the UPDS and DS-LP transitions are close to resonant with a vibrational mode, and splitting of the emission from the UP is observed, consistent with the coherent oscillations in Fig. 3(c). This is reminiscent of the splitting between vibrationally dressed and undressed polaritonic states found in the HTC model [24,66,67]. Finally, for $\Omega_{R}=0.1 \mathrm{eV}$ [Fig. 4(d)], no Rabi splitting is observed, suggesting that the system is in the weak-coupling regime where no polaritons are formed.

To conclude, we have unveiled the temporal dynamics of organic polaritons. We have employed a powerful quasiexact tree tensor network algorithm, which has enabled us to treat a highly structured reservoir of molecular vibrations and free-space emission of the cavity without additional approximations. The simulations reveal coherent vibration-driven oscillations between polaritons and dark states that are only weakly dependent on resonance conditions due to the strong exciton-phonon coupling. This demonstrates the importance of multiphonon processes and non-Markovian dynamics in the system, which are easily underestimated or overestimated in simplified frameworks such as the Bloch-Redfield-Wangsness approximation or the Holstein-Tavis-Cummings model. In addition, the timeresolved emission spectra show fast energy relaxation to the lower polariton, on the scale of tens of femtoseconds. In contrast to Kasha's rule for bare molecules, the radiative and vibrational decays of the upper polariton are similarly fast, and its emission is clearly observed.

This work has been funded by the European Research Council (ERC-2011-AdG-290981 and ERC-2016-STG714870), by the European Union Seventh Framework Programme under Grant Agreement No. FP7-PEOPLE2013-CIG-618229, and the Spanish MINECO under Contract No. MAT2014-53432-C5-5-R and the "María de Maeztu" programme for Units of Excellence in R\&D (MDM-2014-0377). F. A. Y. N.S. and A. W. C. gratefully acknowledge the support of the Winton Programme for the Physics of Sustainability and EPSRC.

*johannes.feist@uam.es

fj.garcia@uam.es
[1] D. G. Lidzey, D. D. C. Bradley, M. S. Skolnick, T. Virgili, S. Walker, and D. M. Whittaker, Strong exciton-photon coupling in an organic semiconductor microcavity, Nature (London) 395, 53 (1998).

[2] J. Bellessa, C. Bonnand, J. C. Plenet, and J. Mugnier, Strong Coupling between Surface Plasmons and Excitons in an Organic Semiconductor, Phys. Rev. Lett. 93, 036404 (2004).

[3] J. Dintinger, S. Klein, F. Bustos, W. L. Barnes, and T. W. Ebbesen, Strong coupling between surface plasmonpolaritons and organic molecules in subwavelength hole arrays, Phys. Rev. B 71, 035424 (2005).

[4] P. Törmä and W. L. Barnes, Strong coupling between surface plasmon polaritons and emitters: A review, Rep. Prog. Phys. 78, 013901 (2015).

[5] D. Sanvitto and S. Kéna-Cohen, The road towards polaritonic devices, Nat. Mater. 15, 1061 (2016).

[6] E. Orgiu, J. George, J. A. Hutchison, E. Devaux, J. F. Dayen, B. Doudin, F. Stellacci, C. Genet, J. Schachenmayer, C. Genes, G. Pupillo, P. Samorì, and T. W. Ebbesen, Conductivity in organic semiconductors hybridized with the vacuum field, Nat. Mater. 14, 1123 (2015).

[7] J. Feist and F. J. Garcia-Vidal, Extraordinary Exciton Conductance Induced by Strong Coupling, Phys. Rev. Lett. 114, 196402 (2015).

[8] J. Schachenmayer, C. Genes, E. Tignone, and G. Pupillo, Cavity-Enhanced Transport of Excitons, Phys. Rev. Lett. 114, 196403 (2015).

[9] J. A. Hutchison, T. Schwartz, C. Genet, E. Devaux, and T. W. Ebbesen, Modifying chemical landscapes by coupling to vacuum fields, Angew. Chem. 124, 1624 (2012).

[10] J. Galego, F. J. Garcia-Vidal, and J. Feist, Cavity-Induced Modifications of Molecular Structure in the StrongCoupling Regime, Phys. Rev. X 5, 041022 (2015).

[11] F. Herrera and F. C. Spano, Cavity-Controlled Chemistry in Molecular Ensembles, Phys. Rev. Lett. 116, 238301 (2016).

[12] K. Bennett, M. Kowalewski, and S. Mukamel, Novel photochemistry of molecular polaritons in optical cavities, Faraday Discuss. 194, 259 (2016).

[13] J. Flick, C. Schäfer, M. Ruggenthaler, H. Appel, and A. Rubio, $A b$ initio optimized effective potentials for real molecules in optical cavities: Photon contributions to the molecular ground state, ACS Photonics 5, 992 (2018).

[14] S. R. K. Rodriguez, J. Feist, M. A. Verschuuren, F. J. GarcíaVidal, and J.G. Rivas, Thermalization and Cooling of Plasmon-Exciton Polaritons: Towards Quantum Condensation, Phys. Rev. Lett. 111, 166802 (2013).

[15] J. D. Plumhof, T. Stöferle, L. Mai, U. Scherf, and R. F. Mahrt, Room-temperature Bose-Einstein condensation of cavity exciton-polaritons in a polymer, Nat. Mater. 13, 247 (2014).

[16] K. S. Daskalakis, S. A. Maier, R. Murray,, and S. KénaCohen, Nonlinear interactions in an organic polariton condensate, Nat. Mater. 13, 271 (2014).

[17] S. Kéna-Cohen and S. R. Forrest, Room-temperature polariton lasing in an organic single-crystal microcavity, Nat. Photonics 4, 371 (2010).

[18] M. Ramezani, A. Halpin, A. I. Fernández-Domínguez, J. Feist, S. R.-K. Rodriguez, F. J. Garcia-Vidal, and J. G. Rivas, Plasmon-exciton-polariton lasing, Optica 4, 31 (2017). 
[19] T. Schwartz, J. A. Hutchison, J. Léonard, C. Genet, S. Haacke, and T. W. Ebbesen, Polariton dynamics under strong light-molecule coupling, Chem. Phys. Chem. 14, 125 (2013).

[20] P. Vasa, W. Wang, R. Pomraenke, M. Lammers, M. Maiuri, C. Manzoni, G. Cerullo, and C. Lienau, Real-time observation of ultrafast rabi oscillations between excitons and plasmons in metal nanostructures with J-aggregates, Nat. Photonics 7, 128 (2013).

[21] E. Eizner, K. Akulov, T. Schwartz, and T. Ellenbogen, Temporal dynamics of localized exciton-polaritons in composite organic-plasmonic metasurfaces, Nano Lett. 17, 7675 (2017).

[22] M. Litinskaya, P. Reineker, and V. M. Agranovich, Fast polariton relaxation in strongly coupled organic microcavities, J. Lumin. 110, 364 (2004).

[23] P. Michetti and G. C. La Rocca, Exciton-phonon scattering and photoexcitation dynamics in J-aggregate microcavities, Phys. Rev. B 79, 035325 (2009).

[24] F. Herrera and F. C. Spano, Theory of nanoscale organic cavities: The essential role of vibration-photon dressed states, ACS Photonics 5, 65 (2018).

[25] M. A. Zeb, P. G. Kirton, and J. Keeling, Exact states and spectra of vibrationally dressed polaritons, ACS Photonics 5, 249 (2018).

[26] J. Prior, A. W. Chin, S. F. Huelga, and M. B. Plenio, Efficient Simulation of Strong System-Environment Interactions, Phys. Rev. Lett. 105, 050404 (2010).

[27] J. Haegeman, J. I. Cirac, T. J. Osborne, I. Pižorn, H. Verschelde, and F. Verstraete, Time-Dependent Variational Principle for Quantum Lattices, Phys. Rev. Lett. 107, 070601 (2011).

[28] A. W. Chin, J. Prior, R. Rosenbach, F. Caycedo-Soler, S. F. Huelga, and M. B. Plenio, The role of non-equilibrium vibrational structures in electronic coherence and recoherence in pigment-protein complexes, Nat. Phys. 9, 113 (2013).

[29] M. L. Wall, A. Safavi-Naini, and A. M. Rey, Simulating generic spin-boson models with matrix product states, Phys. Rev. A 94, 053637 (2016).

[30] F. A. Y. N. Schröder and A. W. Chin, Simulating open quantum dynamics with time-dependent variational matrix product states: Towards microscopic correlation of environment dynamics and reduced system evolution, Phys. Rev. B 93, 075105 (2016).

[31] J. A. Ćwik, S. Reja, P. B. Littlewood, and J. Keeling, Polariton condensation with saturable molecules dressed by vibrational modes, Europhys. Lett. 105, 47009 (2014).

[32] C. Gonzalez-Ballestero, J. Feist, E. Gonzalo Badía, E. Moreno, and F. J. Garcia-Vidal, Uncoupled Dark States Can Inherit Polaritonic Properties, Phys. Rev. Lett. 117, 156402 (2016).

[33] R. Sáez-Blázquez, J. Feist, A. I. Fernández-Domínguez, and F. J. García-Vidal, Enhancing photon correlations through plasmonic strong coupling, Optica 4, 1363 (2017).

[34] N. Christensson, B. Dietzek, A. Yartsev,, and T. Pullerits, Electronic photon echo spectroscopy and vibrations, Vib. Spectrosc. 53, 2 (2010).

[35] Note that the coherent coupling to free-space radiative modes induces a small Lamb shift in the effective cavity frequency that depends on the numerical cutoff frequency $\left(\omega_{r}^{\mathrm{cut}}=3.5 \mathrm{eV}\right.$ in this work).

[36] See Supplemental Material at http://link.aps.org/ supplemental/10.1103/PhysRevLett.121.227401 for details on the chain mapping, tree tensor networks, effects of ensemble size, a comparison with the Holstein-TavisCummings model, and convergence tests, which includes Refs. [37-42].

[37] C. Lanczos, An iteration method for the solution of the eigenvalue problem of linear differential and integral operators, J. Res. Natl. Bur. Stand. 45, 255 (1950).

[38] A. J. Leggett, S. Chakravarty, A. T. Dorsey, M. P. A. Fisher, A. Garg, and W. Zwerger, Dynamics of the dissipative twostate system, Rev. Mod. Phys. 59, 1 (1987).

[39] G. Vidal, Entanglement Renormalization, Phys. Rev. Lett. 99, 220405 (2007).

[40] J. Haegeman, C. Lubich, I. Oseledets, B. Vandereycken, and F. Verstraete, Unifying time evolution and optimization with matrix product states, Phys. Rev. B 94, 165116 (2016).

[41] F. A. Y. N. Schröder, Tensor network states simulations of exciton-phonon quantum dynamics for applications in artificial light-harvesting, Ph.D. thesis, University of Cambridge, 2017.

[42] J. R. Johansson, P. D. Nation, and F. Nori, QuTiP 2: A python framework for the dynamics of open quantum systems, Comput. Phys. Commun. 184, 1234 (2013).

[43] A. W. Chin, S. F. Huelga, and M. B. Plenio, Chain representations of open quantum systems and their numerical simulation with time-adaptive density matrix renormalisation group methods, in Semiconductors and Semimetals (Elsevier Inc., New York, 2011), Vol. 85, p. 115.

[44] U. Schollwöck, The density-matrix renormalization group in the age of matrix product states, Ann. Phys. (Amsterdam) 326, 96 (2011).

[45] S. Szalay, M. Pfeffer, V. Murg, G. Barcza, F. Verstraete, R. Schneider, and Ö. Legeza, Tensor product methods and entanglement optimization for $a b$ initio quantum chemistry, Int. J. Quantum Chem. 115, 1342 (2015).

[46] Y.-Y. Shi, L.-M. Duan, and G. Vidal, Classical simulation of quantum many-body systems with a tree tensor network, Phys. Rev. A 74, 022320 (2006).

[47] C. Guo, A. Weichselbaum, J. von Delft, and M. Vojta, Critical and Strong-Coupling Phases in One- and Two-Bath Spin-Boson Models, Phys. Rev. Lett. 108, 160401 (2012).

[48] F. A. Y. N. Schröder, D. H. P. Turban, A. J. Musser, N. D. M. Hine, and A. W. Chin, Multi-dimensional tensor network simulation of open quantum dynamics in singlet fission, arXiv: 1710.01362.

[49] J. del Pino, F. A. Y. N. Schröder, A. W. Chin, J. Feist, and F. J. Garcia-Vidal, Tensor network simulation of polaronpolaritons in organic microcavities, Phys. Rev. B 98, 165416 (2018).

[50] F. C. Spano, Optical microcavities enhance the exciton coherence length and eliminate vibronic coupling in J-aggregates, J. Chem. Phys. 142, 184707 (2015).

[51] N. Wu, J. Feist, and F. J. Garcia-Vidal, When polarons meet polaritons: Exciton-vibration interactions in organic molecules strongly coupled to confined light fields, Phys. Rev. B 94, 195409 (2016). 
[52] G. Zengin, G. Johansson, P. Johansson, T. J. Antosiewicz, M. Käll, and T. Shegai, Approaching the strong coupling limit in single plasmonic nanorods interacting with J-aggregates, Sci. Rep. 3, 3074 (2013).

[53] R. Chikkaraddy, B. de Nijs, F. Benz, S. J. Barrow, O. A. Scherman, E. Rosta, A. Demetriadou, P. Fox, O. Hess, and J. J. Baumberg, Single-molecule strong coupling at room temperature in plasmonic nanocavities, Nature (London) 535, 127 (2016).

[54] H.-P. Breuer and F. Petruccione, The Theory of Open Quantum Systems (Oxford University Press, New York, 2007).

[55] M. Litinskaya and P. Reineker, Loss of coherence of exciton polaritons in inhomogeneous organic microcavities, Phys. Rev. B 74, 165320 (2006).

[56] R. K. Wangsness and F. Bloch, The dynamical theory of nuclear induction, Phys. Rev. 89, 728 (1953).

[57] A. G. Redfield, Nuclear magnetic resonance saturation and rotary saturation in solids, Phys. Rev. 98, 1787 (1955).

[58] I. Wilson-Rae and A. Imamoğlu, Quantum dot cavity-QED in the presence of strong electron-phonon interactions, Phys. Rev. B 65, 235311 (2002).

[59] D. P. S. McCutcheon and A. Nazir, Quantum dot rabi rotations beyond the weak exciton-phonon coupling regime, New J. Phys. 12, 113042 (2010).

[60] C. Roy and S. Hughes, Phonon-Dressed Mollow Triplet in the Regime of Cavity Quantum Electrodynamics: Excitation-Induced Dephasing and Nonperturbative Cavity Feeding Effects, Phys. Rev. Lett. 106, 247403 (2011).

[61] D. M. Coles, N. Somaschi, P. Michetti, C. Clark, P. G. Lagoudakis, P. G. Savvidis, and D. G. Lidzey, Polaritonmediated energy transfer between organic dyes in a strongly coupled optical microcavity, Nat. Mater. 13, 712 (2014).

[62] J. del Pino, J. Feist, and F. J. Garcia-Vidal, Quantum theory of collective strong coupling of molecular vibrations with a microcavity mode, New J. Phys. 17, 053040 (2015).
[63] T. Neuman and J. Aizpurua, Origin of the asymmetric light emission from molecular exciton-polaritons, arXiv: 1804.08878 .

[64] J. Galego, F. J. Garcia-Vidal, and J. Feist, Suppressing photochemical reactions with quantized light fields, Nat. Commun. 7, 13841 (2016).

[65] J. George, S. Wang, T. Chervy, A. Canaguier-Durand, G. Schaeffer, J.-M. Lehn, J. A. Hutchison, C. Genet, and T. W. Ebbesen, Ultra-strong coupling of molecular materials: Spectroscopy and dynamics, Faraday Discuss. 178, 281 (2015).

[66] F. Herrera and F. C. Spano, Dark Vibronic Polaritons and the Spectroscopy of Organic Microcavities, Phys. Rev. Lett. 118, 223601 (2017)

[67] F. Herrera and F. C. Spano, Absorption and photoluminescence in organic cavity QED, Phys. Rev. A 95, 053867 (2017).

[68] P. G. Savvidis, L. G. Connolly, M. S. Skolnick, D. G. Lidzey, and J. J. Baumberg, Ultrafast polariton dynamics in strongly coupled zinc porphyrin microcavities at room temperature, Phys. Rev. B 74, 113312 (2006).

[69] J.-H. Song, Y. He, A. V. Nurmikko, J. Tischler, and V. Bulovic, Exciton-polariton dynamics in a transparent organic semiconductor microcavity, Phys. Rev. B 69, 235330 (2004).

[70] R. Berera, R. van Grondelle, and J. T. M. Kennis, Ultrafast transient absorption spectroscopy: Principles and application to photosynthetic systems, Photosynth. Res. 101, 105 (2009).

[71] L. Argenti, R. Pazourek, J. Feist, S. Nagele, M. Liertzer, E. Persson, J. Burgdörfer, and E. Lindroth, Photoionization of helium by attosecond pulses: Extraction of spectra from correlated wave functions, Phys. Rev. A 87, 053405 (2013).

[72] S. Baieva, O. Hakamaa, G. Groenhof, T. T. Heikkilä, and J. Jussi Toppari, Dynamics of strongly coupled modes between surface plasmon polaritons and photoactive molecules: The effect of the stokes shift, ACS Photonics 4, 28 (2017). 